\title{
The influence of lightness, and the crispening effect on the per- ceived contrast of textured images
}

\author{
David Kane, Marcelo Bertalmío; Dept. of Information and Communication Technologies, Universitat Pompeu Fabra, Barcelona, \\ Spain
}

\begin{abstract}
We investigate the role of lightness perception in determining the perceived contrast of images. In particular, it is known that the background luminance of a display affects the relationship between onscreen luminance and perceived lightness. Stevens \& Stevens (1963) modeled this effect using a simple power law. However, Whittle (1992) observed a more local effect, whereby subjects are more sensitive to lightness variations around the background luminance (the crispening effect). We probe lightness perception by asking subjects to manipulate the contrast of small patches on a uniform background until they appear to vary, from black to white, in a perceptually linear manner. In a second experiment, we estimate the contrast required to match the contrast of a light patch to that of a dark patch. Both experiments are conducted using five background luminance conditions, from 0 to $100 \%$ luminance. We find that subjects contrast judgments can only be modeled by first estimating the perceived lightness in a scene, using the empirically estimated lightness functions, before computing contrast. We conclude that models of contrast perception must include sophisticated models of lightness perception.
\end{abstract}

\section{Introduction}

The appearance of an image is affected by whether it is viewed with a light or a dark surround [7]. It is well established that the surround luminance of stimulus affects the manner in which the onscreen luminance levels are perceived [4]. It is also found that the surround luminance affects the perceived contrast of a stimulus [1]. Indeed most models of contrast perception pass the stimulus through a luminance nonlinearity prior to computing the image contrast and it has been demonstrated that an adaptive model of the luminance nonlinearity is required before accurate, surround independent estimates of contrast can be made [9].

Under some conditions, the luminance nonlinearity may be modeled using a simple power-law [13,3]. For instance the influential CIECAM02 uses an adaptive power-law with an exponent that adapts and becomes more compressive when the surrounding luminance values are darker [11]. However, under some circumstances the function becomes considerably more complex. In particular, Whittle [14] observed an effect whereby luminance judgments are more sensitive around the background luminance, and in turn, judgments of luminance are biased away from the background luminance. Whittle termed this the 'crispening' effect. Functions that include the 'crispening' effect are not well modeled by a simple power-law as shown in Figure 2.

The role of 'crispening' in determining the perception of real world images is unknown. Although the 'crispening' effect has been observed when using simple, artificial stimuli, the effect was not observed when the perceived luminance of real world scenes was investigated [4]. Moreover, Whittle [14] observed that the effect is abolished when a small annulus separates the background luminance from the test stimuli. Thus there is reason to think the effect may only play a minimal role in the perception of natural scenes, perhaps enhancing contrast between image boundaries. Nonetheless the role of 'crispening' remains to be established.

The aim of this paper is to evaluate the relationship between lightness- and contrast- perception. The paper is an extension upon [9], which revealed that judgments of image quality are highly correlated with a simple model of contrast perception, detailed in the next section. The study examined quality judgments for small real world images presented on a uniform black, gray or white background. The results demonstrated that an adaptive luminance nonlinearity was required to accurately predict the image quality scores. The luminance nonlinearity was assumed to model lightness perception, however this assumption was not directly tested. Accordingly, in this study we wish to develop a research paradigm that will allow us to directly study both phenomena using stimuli that are as similar as possible. The aim is to establish to what extent does luminance adaptation affect contrast thresholds. In this regard, there are many potential unresolved questions, for instance: Does contrast perception relate to lightness perception (as presumed above), or does brightness perception provide a better correlate? Does anchoring theory [8] play a role in the perception of contrast? In this study we shall focus on the role of 'crispening' upon contrast judgments. In the first experiment we examine perceived lightness by asking subjects to manipulate the luminance of small patches until they appear to transition, in a linear manner, from black to white. In a second experiment, using a very similar experimental set up, we examine the perceived contrast of image textures with different mean luminance values. Both experiments are conducted with five background luminance levels and with and without a small contrast annulus.

\section{Contrast model}

This work directly extends the simple contrast model of [9] by using the empirically estimated lightness functions obtained in experiment one. Thus perceived lightness $L$ is some unknown function of onscreen luminance $I$ :

$$
L=F(I)
$$

Second, the standard deviation of the 'lightness' image is computed:

$$
\sigma=\sqrt{\frac{1}{N} \sum_{i=1}^{N}\left(L_{i}-\bar{L}\right)^{2}}
$$



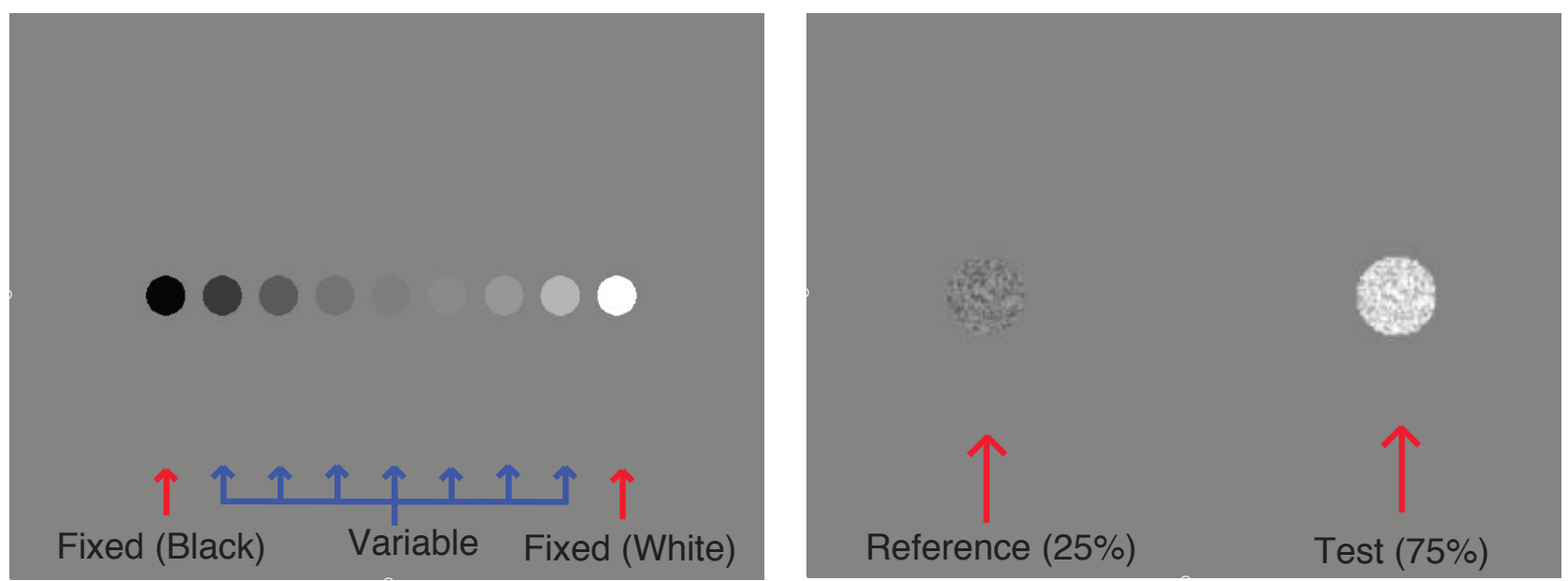

Figure 1. Left: Screen shot from the lightness experiment after the luminance values have been adjusted to achieve perceptual linearization. Right: Clipped screenshot of the contrast experiment after the stimulus have been matches for perceived contrast.

Finally, the third stage is a simple power-law non-linearity with a fixed exponent of 0.35 to produce $C$, the estimate of stimulus contrast:

$$
C=\sigma^{0.35}
$$

\section{Methods Subjects}

Two subjects completed both experiments. Both had corrected to normal vision. One was an author of the paper. Ethics was approved by the Comité Etico de Investigacion Clinica, Parc de Salut MAR, Barcelona, Spain and all procedures complied with the declaration of Helsinki.

\section{Apparatus}

Both experiments were conducted on a Philips 109B CRT monitor with spatial and temporal resolutions of 1280 by 960 pixels and $75 \mathrm{~Hz}$. The display was viewed at a distance of $58 \mathrm{~cm}$ so that 36 pixels subtended 2 degree of visual angle. The full display subtended 35.5 by 25.5 degrees. The monitors minimum luminance was $0.6 \mathrm{cdm}^{-2}$ and the maximum $80 \mathrm{cdm}^{-2}$. The decoding nonlinearity of the CRT monitor was recored using a Konica Minolta LS 100 photometer. Stimuli were generated on an Apple MacBook running MATLAB (MathWorks) with functions from the Psychtoolbox [5, 12].

\section{Stimuli}

Experiment one: Subjects viewed 9 circular patches all with a 2 degree radius. In all cases we shall describe luminance in terms of the percentage of maximum luminance. The leftmost circle had a fixed luminance of $0 \%$, the rightmost circle has a fixed luminance of $100 \%$. The initial starting luminance of the 7 intermediary circles was randomized. The fifth (middle) circle was presented in the center of the monitor and each circle was separated by one degree.

Experiment two: Subjects viewed 2 circular patches both with a 2 degree radius. The stimulus were presented along the center of the vertical axis and the two circles were displayed at the $3 / 8$ and 5/8 horizonal positions. The patches were composed of white noise that was resampled to four times the initial area using Matlab's imresize using bicubic interpolation. Thus the signal was blurred and low-pass. This was chosen to increase the perceptual visibility of the white-noise stimuli.

\section{Monitor output}

Experiment one: Experiment one uses a maximum of ten luminance values. To achieve a continuous, quantization-free output, the color look up table (CLUT) was updated on each frame to produce the output voltage corresponding to the desired luminance (taking into account the encoding nonlinearity of the monitor).

Experiment two: The stimuli used in experiment two was continuous. Accordingly, a fixed CLUT table corresponding to the inverse of the monitor's decoding nonlinearity was used. No technique was used to increase the effective bit-rate of the monitor.

\section{Procedure}

Experiment one: Subjects were asked to adjust the luminance of seven circles until the appeared to transition, from black to white, and from left to right, in a perceptually linear manner. Subjects adjusted the luminance of the patch via a key press. Five keys were in use; the left and right buttons allowed for luminance translations of $0.01 \%$, while the up and down keys allowed for a transition of $1 \%$. Continued key presses allowed preformed a continual luminance translation until either the maximum or minimum luminance was reached which correspond in all conditions to the minimum and maximum luminance of the monitor. The space key was pressed when the subjects had completed the task to their satisfaction and the next background luminance condition was shown.

Experiment two: Subjects were asked to select the image patch with the greater contrast. Answers were indicated by pressing the corresponding left or the right arrow-keys. The position of the test and reference patch was randomized on each trial. The method of contrast stimuli was used to alter the contrast of the test patch and a cumulative gaussian was fit to the data to find the point-of-subjective equality (PSE). 


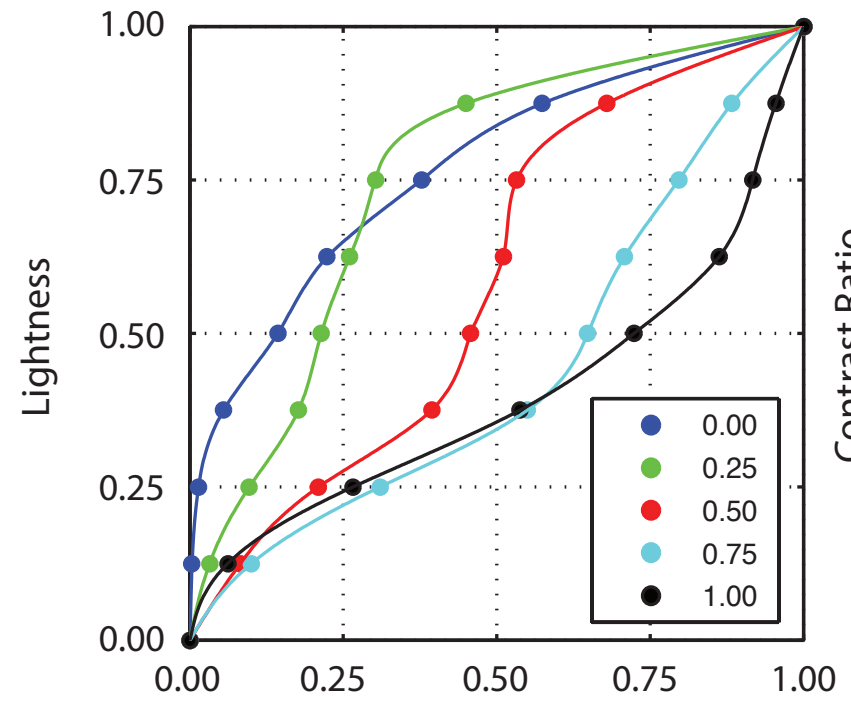

Luminance

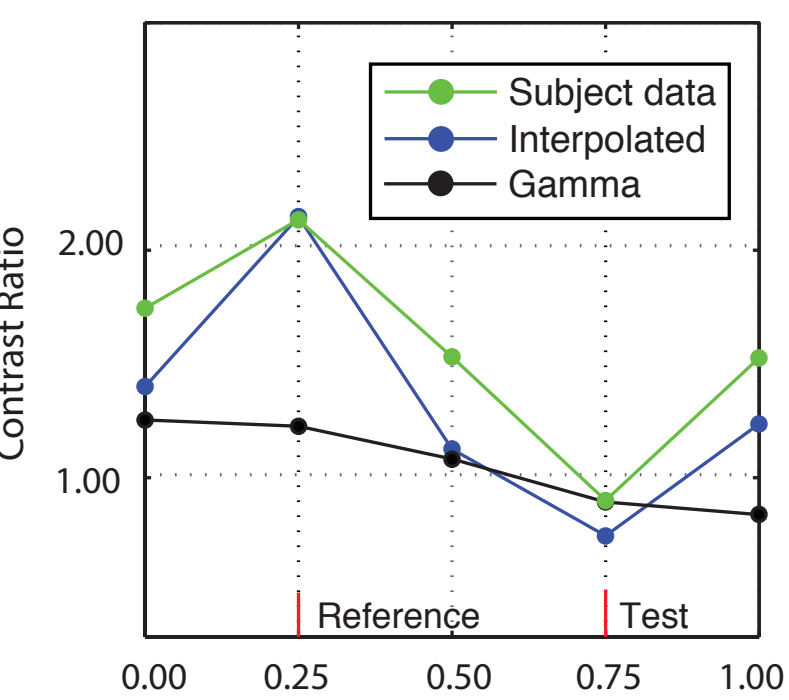

Background Luminance

Figure 2. Left: The onscreen luminance to lightness functions obtained for five background luminance conditions and no annulus. Right: The green data indicates the ratio of contrast needed to match the lighter image patch with the darker image patch as a function of the background luminance level. See text for details on the model performance denoted by the blue and black lines.

\section{Experimental Results}

\section{Lightness}

The results of the lightness and contrast experiments for the no-annulus condition are shown in Figure 2. The lefthand graph plots perceived lightness as a function of onscreen luminance for the five different background luminance levels as denoted by the legend. Note, in this figure the lightness judgments are at equal steps on the ordinate, and the abscissa is varied according to the mean luminance value set by our subjects. The results show that, in a crude sense, the functions become less compressive as the background luminance is increased. The functions also demonstrate a clear evidence of a 'crispening' effect whereby the lightness judgements become biased away from the background luminance levels.

\section{Contrast}

The results of the contrast experiment in the no-annulus conditions are shown on the righthand graph. In this figure, the reference patch always has a mean luminance of $25 \%$ and the test patch a mean luminance of $75 \%$. The contrast of the lighter test patch was adjusted until the point-of-subjective-equality (PSE) was found. The ordinate plots the ratio of the contrast of the test over the reference patch as a function of the background luminance level of the stimulus. The green data denotes the subject data. The function has a clear peak and trough, with the darker patch perceived to have roughly twice the contrast when the background luminance is matched. In contrast, when the background luminance is matched to the background luminance, the patches are perceived to have roughly equal contrast.

\section{Model}

We investigate whether the empirically estimated lightness functions are required to predict the contrast data. To do so, we either fit a simple power law to the lightness functions by finding the best-fitting exponent, or generate an interpolated functions via Matlab's interp function. This procedure produces a smoothed, up-sampled function. This procedure constrains the interpolated function to pass through the original data points. The function was then used as a look-up table to generate a finely quantized (12bit) estimate of the perceived lightness of the test and reference contrast patches. Thus either the best fitting power-law or the lookup table was used as the function described in equation 1 that relates onscreen luminance to perceived lightness. In this manner estimates of contrast for the darker and lighter image patches may be obtained and the associated contrast ratio computed. The results shown Figure 2 by the blue and black lines, demonstrate that modeling lightness as a simple power-law (black) is unable to predict the data. In contrast, the empirically estimated lightness functions produce contrast estimates (blue) that both predict the overall shape, and the magnitude of the subjects' contrast ratios.

\section{Additional test and reference mean luminance combina- tions}

To provide a further test of the model we extend the contrast experiment to different combinations for the mean luminance of the test and reference contrast patches. The results are shown in figure 3. Different colors denote different reference-test combinations and the solid and dashed lined the subject and model data respectively. Encouragingly the model is able to capture the data in the additional conditions. 


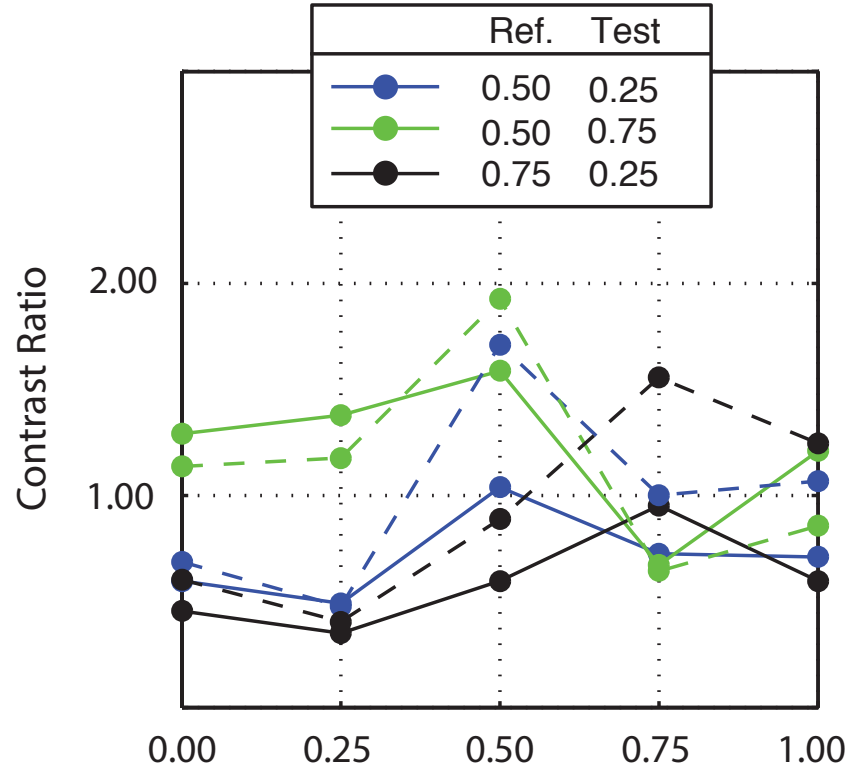

Background Luminance

Figure 3. Results from the contrast experiment using different combinations of reference and test stimuli mean luminance as denoted by the legend. Solid lines indicates the subject data. Dashed lines indicated the model data.

\section{No annulus}

Whittle [14] observed that the 'crispening' effect could be abolished by the addition of an annulus that separated the luminance of the test patches from the background luminance. Accordingly we apply a small, but high contrast annulus to the stimuli in both the lightness and the contrast experiments. In this case the annulus is salt and pepper, maximal contrast white noise with a width of 15 acrmin. The results shown in Figure 2 demonstrate that when the annulus is introduced, the 'crispening' effect is nearly abolished and the functions are now more closely approximated by a simple power-law. The results in the contrast experiment demonstrate that with the addition of the annulus, the distinct peak and trough of the contrast ratios have now disappeared and the darker stimulus is always perceived as having a higher contrast. The results of the model, again using the empirically estimated lightness functions, capture the basic shape of the contrast ratios, but cannot capture the magnitude. Instead the model predicts that the perceived contrast of the dark and light patches is more equal than the subjects contrast ratios demonstrate.

\section{Conclusion}

The aim of the study is develop a paradigm that allows us to directly investigate how the luminance nonlinearity of the human visual system affects contrast perception. The close relationship between the model of contrast perception, using the empirically estimated lightness functions, and the subjects' data indicate that (a) the luminance nonlinearity and contrast perceptions are indeed closely intertwined, (b) that 'crispening' does play a substantial role in determining the contrast of textures and (c) that the current research paradigm is suitable for the task at hand. We hope that future studies using this paradigm will begin to reveal the true role of 'crispening' in determining the appearance of real world images.

We note several difficulties with the current study however. First, the so-called lightness functions obtained in experiment one are noisy and subjects reported that they did not like the task. Given the importance of the shape of the resulting functions to determining contrast, it is important the data obtained is reliable. One problem with the experiment is that an alteration to one luminance level may require alterations to all the other luminance levels and this can be frustrating for subjects. A second, potential problem is that when nine circles are used, the transitions are always substantially supra-threshold and it may be tricky judging the magnitude of supra-threshold transitions. Arguably, using more circles may make the task easier (Whittle used 24). Whittle also demonstrated [14] that there is a close relationship between sensitivity to luminance variations and supra-threshold lightness perception. Threshold judgments are less prone to bias than supra threshold judgments [10], thus they may provide a more reliably means of determining the luminance nonlinearity. An alternative approach is to establish how reliable the observed nonlinearities are across different experimental conditions. It might be that the functions derived in the original study by Whittle are suitable for the current study.

The predicted and subjects' contrast thresholds exhibit a very similar pattern in the no-annulus conditions. However, the correspondence between the model and subjects' data is less convincing in the with-annulus condition. Specifically, subjects perceive the darker stimulus to have substantially higher contrast than the model predicts. It is unclear at this stage whether this indicates that the lightness functions do not always predict the subjects data or whether another unknown factor is at play. One potential factor is contrast gain control [6] which will be stronger in the presence of the annulus, although this does not directly predict a higher perceived contrast for the darker stimulus. An equiluminant surround that does not introduce a luminance contrast signal could be used to investigate the role of contrast gain. Ongoing investigations indicate that the spatial frequency profile of the textures may also play a substantial role in determining perceived contrast. Specifically, patches with a higher mean luminance are perceived with higher contrast when the stimulus has a higher spatial frequencies and vice-versa. This pattern may reflect the impact of the absolute luminance value on the contrast sensitivity function for which the peak of sensitivity moves to higher spatial frequencies with increasing luminance [2] and indicates that spatial frequency processing will need to be incorporated into the model.

\section{References}

[1] Peter GJ Barten. Evaluation of subjective image quality with the square-root integral method. JOSA A, 7(10):2024-2031, 1990.

[2] Peter GJ Barten. Formula for the contrast sensitivity of the human eye. In Electronic Imaging 2004, pages 231-238. International Society for Optics and Photonics, 2003.

[3] CJ Bartleson. Optimum image tone reproduction. Journal of the SMPTE, 84(8):613-618, 1975.

[4] CJ Bartleson and EJ Breneman. Brightness perception in complex fields. Josa, 57(7):953-956, 1967. 


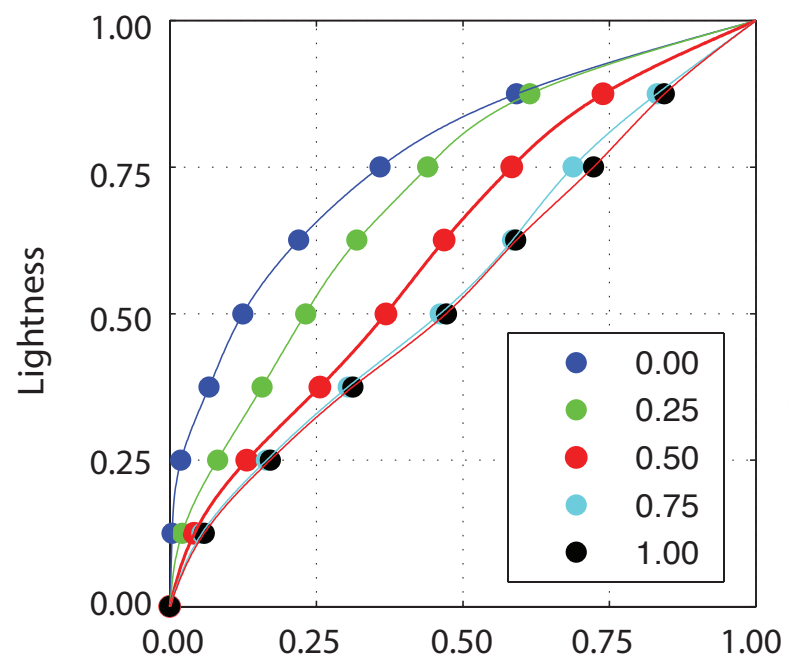

Luminance

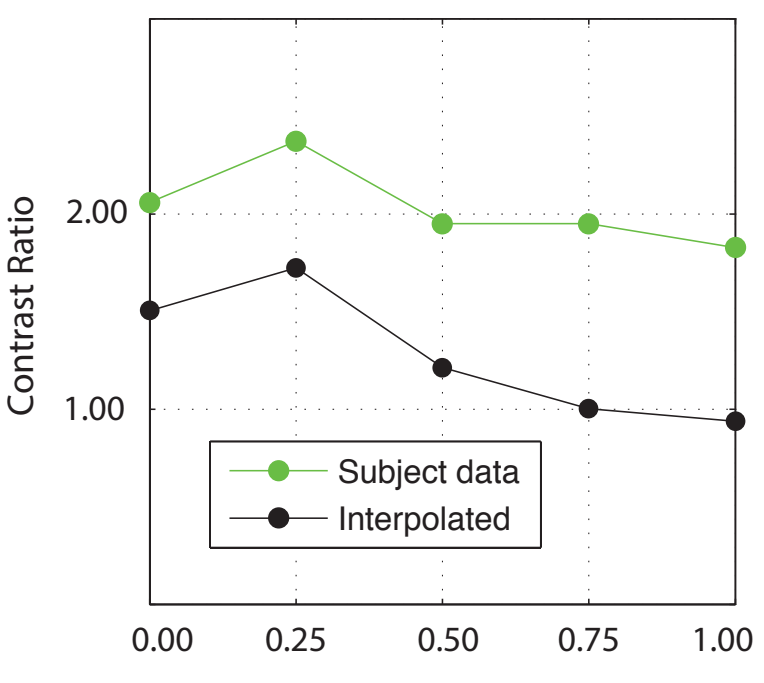

Background Luminance

Figure 4. When a small contrast annulus is added to the stimulus the lightness functions do not exhibit the same 'crispening' effect observed without the annulus. Likewise, the contrast data does not exhibit the same peak and trough as a function of the background luminance.

[5] David H Brainard. The psychophysics toolbox. Spatial vision, 10:433-436, 1997.

[6] Matteo Carandini, David J Heeger, and J Anthony Movshon. Linearity and normalization in simple cells of the macaque primary visual cortex. The Journal of Neuroscience, 17(21):8621-8644, 1997.

[7] Mark D Fairchild. Considering the surround in deviceindependent color imaging. Color Research and Application, 20(6):352-363, 1995.

[8] Alan Gilchrist, Christos Kossyfidis, Frederick Bonato, Tiziano Agostini, Joseph Cataliotti, Xiaojun Li, Branka Spehar, Vidal Annan, and Elias Economou. An anchoring theory of lightness perception. Psychological review, 106(4):795, 1999.

[9] David Kane and Marcelo Bertalmío. The role of lightness perception in determining the perceived contrast of real world scenes. In Proc. IS\&T International Symposium on Electronic Imaging, 2016.

[10] Michael J Morgan, D Melmoth, and Joshua A Solomon. Linking hypotheses underlying class a and class b methods. Visual neuroscience, 30(5-6):197-206, 2013.

[11] N. Moroney, M.D. Fairchild, R. Hunt, C. Li, M.R. Luo, and T. Newman. The CIECAM02 color appearance model. In Proceedings of Color and Imaging Conference. Society for Imaging Science and Technology, 2002.

[12] Denis G Pelli. The videotoolbox software for visual psychophysics: Transforming numbers into movies. Spatial vision, 10(4):437-442, 1997.

[13] S.S. Stevens. To honor Fechner and repeal his law. Science, 133:80-133, 1961.

[14] Paul Whittle. Brightness, discriminability and the crispening effect. Vision research, 32(8):1493-1507, 1992.

\section{Author Biography}

David Kane received his PhD from University Collage London where he studied under Steven Dakin and Peter Bex and learnt the basics of low-level psychophysics. From there David chose to apply his skills at the intersection of perception and technology and took a position working under Professor Marty Banks at The University of California Berkeley investigating issues surrounding the development of stereo $3 D$ displays. Currently, David is working as in-house experimental psychologist in the image processing lab Marcelo Bertamlío at Universitat Pompeu Fabra.

Marcelo Bertalmío received the Ph.D. degree in electrical and computer engineering from the University of Minnesota in 2001. He is an Associate Professor at University Pompeu Fabra in Barcelona, Spain. His interests are Image Processing and Computer Vision for digital cinema applications, although he prefers the (analog) films of Preston Sturges and Luis Buũel. 